\title{
Impurity position effect on the diamagnetic susceptibility of a magneto-donor in GaAs inhomogeneous quantum dots
}

\author{
Sanae Janati Edrissi ${ }^{1}$, Ali Mmadi ${ }^{1,2}$, Izeddine Zorkani ${ }^{1 *}$, Khalid Rahmani $^{3}$, Anouar Jorio ${ }^{1}$ Mohamed El Ganaoui ${ }^{4}$ \\ ${ }^{1}$ University Sidi Mohamed Ben Abdellah, Laboratory of Solid State Physics, Faculty of Sciences Dhar El Mahraz, Group of \\ Nanomaterial and Renewable Energies, Fez, Morocco \\ ${ }^{2}$ Faculty of Sciences and Techniques-University of Comores, B.P 2585, Moroni Comoros \\ ${ }^{3}$ LIRST, Faculty Polydisciplinary, Béni Mellal -Morocco \\ ${ }^{4}$ LERMAB, IUT de Longwy, University of Lorraine 186, rue de Lorraine. F- 54400 Cosnes et Romain, France
}

\begin{abstract}
Inhomogeneous Quantum Dot (IQD) semiconductor represents a newest trend in condensed matter, due to their important quantum levels and the outstanding properties. In this work, the impurity position effect on the diamagnetic susceptibility of a shallow magneto-donor, confined to move in (IQD) made out of $\mathrm{Ga}_{1-\mathrm{x}} \mathrm{Al}_{\mathrm{x}} \mathrm{As} / \mathrm{GaAs} / \mathrm{Ga}_{1-\mathrm{x}} \mathrm{Al}_{\mathrm{x}} \mathrm{As}$ is reported theoretically. With the increase of the magnetic field, the diamagnetic susceptibility increases. The results using variational method reveal that diamagnetic susceptibility depends on many parameters including the impurity position, the external magnetic field and the nanostructure size. The magnetic field effect is more pronounced when the donor is placed near the extremities of the spherical layer (off-center). In addition, a maximum of diamagnetic susceptibility is observed, corresponding to a critical position value, for strong confinement regime and when the impurity is located in the spherical layer center.
\end{abstract}

\section{Introduction}

Semiconductors play a vital role in the industry and many semiconductor-based electronic devices are present in our daily lives: desktops, tablets, smartphones, flat screen and sensors [1].This is the result of fundamental studies that have made it possible to understand the properties of semiconductors, such as band structure, transport properties or photoluminescence, and more applied studies that have taken place over many decades.

On the other hand, the industrial processes development for designing semiconductors on the form of nanoscale is one of the main challenges in nanotechnology to obtain platforms for producing quantum nanostructures with low-dimensional such as Quantum Wells, Quantum Well Wires and Dot by direct epitaxial growth [2,3].

The interest in these systems resides essentially in the possibility of various device applications and the studies on these systems have been extensively reported in the literature [4-6]. Recently a new class of spherical quantum dots called Inhomogeneous QD or Quantum Dot-Quantum Well (QDQW) composed of two semiconductor materials have been fabricated and studied. Among this category, the quantum dots with the smaller bulk band gap are located between a core and outer shell of the material with the larger band gap. With the progress synthesis and fabrication of different nanomaterials various kinds of tubular structures such as $\mathrm{CdS} / \mathrm{HgS} / \mathrm{CdS}$ [7-8], CdSe/ZnS/CdSe [9] and $\mathrm{Ga}_{1-\mathrm{x}} \mathrm{Al}_{\mathrm{x}} \mathrm{As}$ / GaAs/ $\mathrm{Ga}_{1-\mathrm{x}} \mathrm{Al}_{\mathrm{x}} \mathrm{As}$ [10] and others, are obtained and

\footnotetext{
${ }^{*}$ Corresponding author: izorkani@hotmail.com
}

attract much attention. The effect of an applied external field on the physical properties of such structures has been reported experimentally and theoretically. In references [11-13], the ground state energy of an offcenter donor and binding energy of excitons in inhomogeneous quantum dots under uniform electric field and magnetic field have been investigated. Recently, the effects of impurity position and magnetic field on the shallow-donor binding energy in $\mathrm{GaN}($ core)/InGaN(well)/GaN (shell) have studied by El Ghazi and coworkers [14].Their results revealed that the binding energy depends strongly on the external magnetic field, the impurity position and the structure radius. Moreover, one of the most recent works in this field was in 2011 by Rahmani and coworkers [15] who gave an estimate of binding energy and diamagnetic susceptibility for a donor impurity. These quantities depend on the radius of the heart and the shell of the QDQW therefore of the ratio inner radius (of the heart) by the outer radius (of the shell). The authors have shown that for a critical value of this ratio, the binding energy has a minimum. In addition, the binding energy and the diamagnetic susceptibility depend on the position of the donor. The susceptibility is maximum if the impurity is placed in the center of the spherical layer. Ogli and Rostami [16] investigated the photoluminescence intensity of the QDQW heteronaostructure by adopting a model of nonlinear core potential that was analyzed by numerical finite element method and compared with thes traditional linear potential. These authors probed the effect of charge carrier localization on photoluminescence 
intensity. In addition, the study focused on the dependence of the photoluminescence intensity as a function of the radius of the core material, the thicknesses of the well material and the outer shell material. The authors demonstrated the shift of the emission wavelength in the QDQW by the introduction of the nonlinear core potential which contributed significantly in the biological applications

The magnetic field effects on the diamagnetic susceptibility and binding energy of a hydrogenic impurity in a QWW and in cylindrical quantum dot CQD have been investigated by Mmadi and coworkers [17].Their results show that the diamagnetic susceptibility is more important for donors in QWW and CQD over a large dimension structure. In a previous paper, we have investigated the diamagnetic susceptibility of a confined donor in GaAs Inhomogenous Quantum Dot with and without magnetic field [18]. We have found that the diamagnetic susceptibility increases with the magnetic field and it is more important especial for larger quantum dot. We have demonstrated that the diamagnetic susceptibility shows a minimum for a critical value of the ratio $R_{1} / R_{2}$, depending on the value of the outer radius. In our previous work we have studied the effect of the shape on the diamagnetic susceptibility and binding energy of a donor Confined in a Spherical Quantum Dot (SQD) and Cylindrical Quantum Dot (CQD) [19].

Generally, theoretical or experimental studies on the impurity position effect on the diamagnetic susceptibility of a magneto-donor placed in an IQD have not been reported. In the present work, we use a variational method to calculate the Diamagnetic Susceptibility in a $\mathrm{Ga}_{1-\mathrm{x}} \mathrm{Al}_{\mathrm{x}} \mathrm{As} / \mathrm{GaAs} / \mathrm{Ga}_{1-\mathrm{x}} \mathrm{Al}_{\mathrm{x}} \mathrm{As}$ IQD in presence of a magnetic field in order to further improve the optical properties of inhomogeneous quantum dots. This paper is organized as follows: in Section 2 we explain the Hamiltonian of hydrogenic impurity ground state in the presence of a magnetic field, we deduce the expression of the magneto donor Diamagnetic Susceptibility. The numerical results and conclusion are presented in Section 3.

\section{Model and Theory}

Our material based on a Quantum Well-Quantum Dot nanostructure composed of three spherical semiconductor layers subjected to the action of a uniform magnetic field $\vec{B}$ and parallel to the axis $\mathrm{Oz}$ (see Figure 1). As an illustration, we will take the structure of $\left[\mathrm{Ga}_{1-\mathrm{x}} \mathrm{Al}_{\mathrm{x}} \mathrm{As}\right.$ (Core)/GaAs (Well)/ $\mathrm{Ga}_{1-\mathrm{x}} \mathrm{Al}_{\mathrm{x}} \mathrm{As}$ (Shell)] $R_{1}$ and $R_{2}$, respectively, denote the inner and outer radius of the IQD.

In the framework of the approximation of the effective mass, the Hamiltonian describing the interaction of an electron with a hydrogenic impurity placed at the position $\mathrm{a}_{0}$ in an IQD is given by:

\footnotetext{
${ }^{*}$ Corresponding author: izorkani@hotmail.com
}

$H=\frac{1}{2 m^{*}}\left(\vec{P}-\frac{e}{c} \vec{A}\right)^{2}-\frac{e^{2}}{\varepsilon_{0}\left|\vec{r}-\vec{a}_{0}\right|}+V_{\text {conf }}(r)$

Where $\varepsilon_{0}$ is the static dielectric constant of the material, $m^{*}$ is the effective electron mass and $\vec{A}$ is the magnetic field potential. We consider a homogeneous magnetic field along the z-axis, and the vector potential is given by: $\vec{A}=\frac{1}{2} \vec{B} \times \vec{r}$.

In the present work we consider an infinitely deep well:

$V_{\text {conf }}(\vec{r})=\left\{\begin{array}{cccc}0 & R_{1}< & r< & R_{2} \\ \infty & r<R_{1} & \text { and } & r>R_{2}\end{array}\right.$

The Hamiltonian for the ground state, in spherical coordinates, can be expressed as:

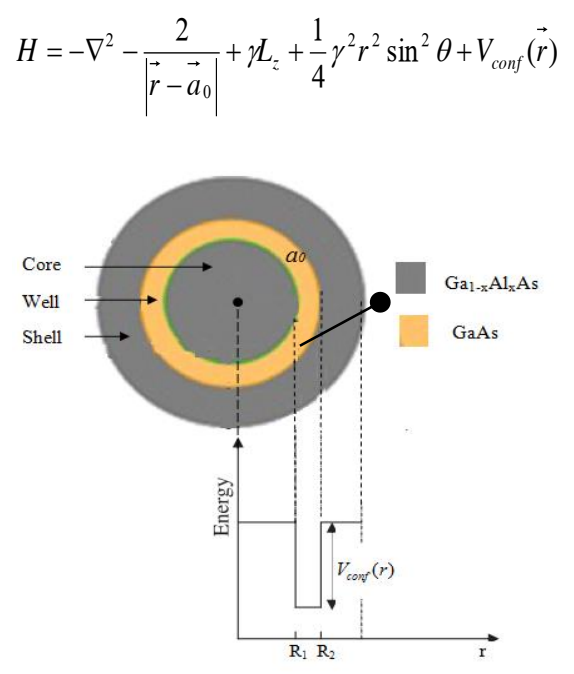

Fig. 1. A schematic graph of the spherical IQD. The impurity position is a 0 where $\mathrm{R} 1$ and $\mathrm{R} 2$ are the inner and the outer radius respectively.

Where $\left|\vec{r}-\vec{a}_{0}\right|=\sqrt{r^{2}+a_{0}^{2}-2 r a_{0} \operatorname{Cos} \theta}$ is the distance between the electron and the impurity site. We use the effective Bohr radius $a^{*}=\frac{\hbar^{2} \varepsilon_{0}}{m^{*} e^{2}} \quad$ and the effective Rydberg $R^{*}=\frac{m^{*} e^{4}}{2 \hbar^{2} \varepsilon_{0}^{2}} \quad$ as the units of length and energy. We introduce the dimensionless parameter $\gamma=\frac{\hbar \omega_{c}}{R^{*}}$ characterizing the strength of the magnetic field where $\omega_{c}=\frac{e B}{m^{*} c}$ is the effective 
cyclotron frequency. We use a variational method approach to determine the ground state binding energy; we adopt the wave function given in Ref [14]:

$$
\psi=\frac{\operatorname{Sin}\left[\pi /\left(R_{2}-R_{1}\right)\left(r-R_{1}\right)\right]}{r} \exp \left(-\lambda \sqrt{r^{2}+a_{0}^{2}-2 r a_{0} \operatorname{Cos} \theta}\right)
$$

Where $\lambda$ is a variational parameter . The exponential factor describes the Coulomb spatial interaction. The ground-state energy of the system is given by:

$$
E=\min _{\lambda}\left\{\frac{\langle\psi|\mathrm{H}| \psi\rangle}{\langle\psi \mid \psi\rangle}\right\}
$$

And the binding energy $E_{b}$ of the hydrogenoide donor impurity is defined as the difference between the energy of the electron in the absence $\left\langle H_{0}\right\rangle$ and in the presence of the impurity $\min _{\lambda}\langle H\rangle\left(\mathrm{H}_{0}\right.$ is the Hamiltonien in the absence of the impurity).

$$
\mathrm{E}_{b}=\left\langle H_{0}\right\rangle-\min _{\lambda}\langle H\rangle
$$

The expression of the diamagnetic susceptibility of the donor impurity, $\chi_{\text {dia }}$ in atomic unit (a.u), is given by [20]:

$\chi_{\text {dia }}=-\frac{e^{2}}{6 m^{*} \varepsilon_{0} c^{2}}<\left(r-a_{0}\right)^{2}>$

Where $\mathrm{c}$ is the velocity of light and $\left\langle\left(r-a_{0}\right)^{2}>\right.$ is the mean square distance of the electron from the nucleus. The diamagnetic susceptibility is the capacity that negative charges tend to form screen between the inside of the body and the applied magnetic field. The final result for the impurity position effect in presence of a magnetic field is obtained by numerical minimization of the groundstate energy of the system with the impurity respect to the variational parameter $\lambda$.

\section{Numerical results and Discussion}

We will present our results for the cases an IQD made out of $\mathrm{Ga}_{1-\mathrm{x}} \mathrm{Al}_{\mathrm{x}} \mathrm{As} / \mathrm{GaAs} / \mathrm{Ga}_{1-\mathrm{x}} \mathrm{Al}_{\mathrm{x}} \mathrm{As}$. We will consider two extreme configurations: the first is stated for $\mathrm{R}_{1}=0$ and corresponds to the Homogenous quantum dot "HQD" of radius $\mathrm{R}_{2}$; and the second corresponds the IQD where $\mathrm{R}_{1}$ tends to $\mathrm{R}_{2}$, for $\mathrm{R}_{2}$ fixed (which corresponds to an infinitely thin spherical layer).

\footnotetext{
* Corresponding author: izorkani@hotmail.com
}

The diamagnetic susceptibility $\chi_{\text {dia }}$ versus the HQD radius $R_{2}$ for different values of the donor position $\mathrm{a}_{0} / \mathrm{R}_{2}=0,3 / 4$ and 1 are presented in Figure 2.We see that the diamagnetic susceptibility $\square_{\text {dia }}$ decreases as the donor position increases for different value of $\mathrm{a}_{0} / \mathrm{R}_{2}$ (when the donor moves from the center to the surface of the QD). Our results show that for strong confinement the diamagnetic susceptibility decreases and tends to the Quantum Well value. Nevertheless, for weak confinement the diamagnetic susceptibility decreases with the increase of HQD radius and approaches to the three dimensional value which correspond to the Bulk limit case. Our results are in good agreement with previous calculations without the magnetic field and with the work of Mmadi and coworkers [18].

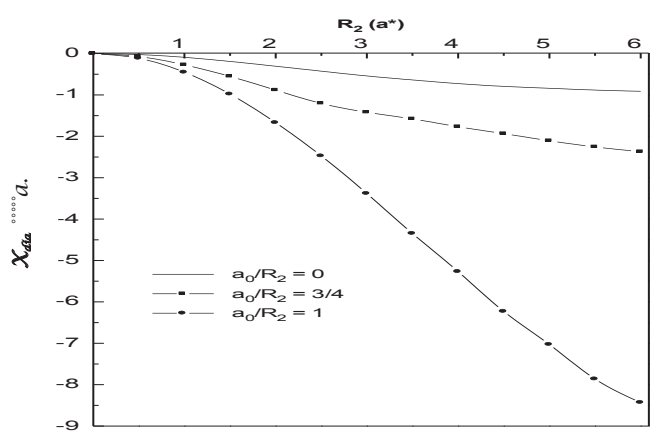

Fig. 2. The Diamagnetic Susceptibility in the absence of the magnetic field as a function of the HQD radius R2 for different values of the donor position $\mathrm{a} 0 / \mathrm{R} 2=0, \mathrm{a} 0 / \mathrm{R} 2$ $=3 / 4$ and $\mathrm{a} 0 / \mathrm{R} 2=1$.

The diamagnetic susceptibility $\chi_{\text {dia }}$ are presented in Figure 3 in the absence of magnetic field as a function of the impurity position for three values of the IQD inner radius $\left(\mathrm{R}_{1}=0.5 \mathrm{a}^{*}, 1 \mathrm{a}^{*}\right.$ and $\left.2 \mathrm{a}^{*}\right)$ with $\mathrm{R}_{2}=3 \mathrm{a}^{*}$. We notice that the diamagnetic susceptibility as a function of the impurity position increase reach a maximum and the decrease as $a_{0}$ increase from 0.5 to $3 a^{*}$. The position of this maximum is characterized by the inner and the outer radius (It's value is around $\mathrm{a}_{0}=2 \mathrm{a} *$ for $\left(\mathrm{R}_{1}=1 \mathrm{a} *\right.$ and $\left.\mathrm{R}_{2}=3 \mathrm{a} *\right)$ for example). This result shows that the maximum of the diamagnetic susceptibility $\chi_{\text {dia }}$ is obtained when the donor impurity is equation to $a_{0}=\left(R_{1}+R_{2}\right) / 2$. This result is in good agreement to the one obtained in ref [14]. At this position the orbital electronic presents the spherical symmetry, the diamagnetic susceptibility is maximal and decreases when the donor moves toward the extremities for larger dimension of the IQD. 


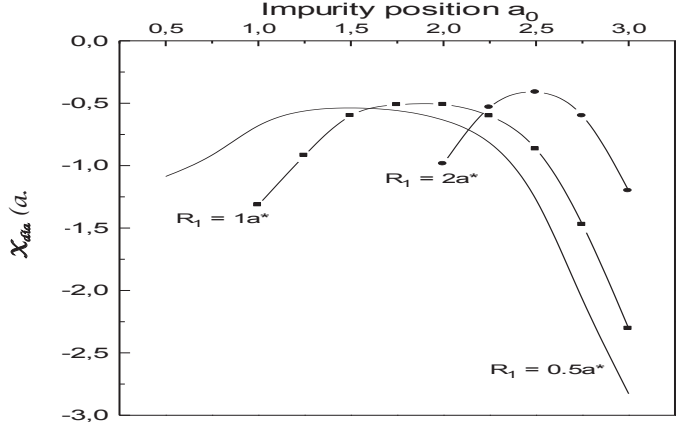

Fig. 3.Diamagnetic Susceptibility $\square$ in the absence of magnetic field as a function of the impurity position for different values of the inner radius of the SQDQW: R1 = $0.5 \mathrm{a}^{*}, 1 \mathrm{a}^{*}$ and $2 \mathrm{a}^{*}$. with $\mathrm{R} 2=3 \mathrm{a}^{*}$

We present in Figure 4, the diamagnetic susceptibility $\chi_{\text {dia }}$ as a function of $\mathrm{R}_{1} / \mathrm{R}_{2}$ for two confinement regimes $\left(\mathrm{R}_{2}=1 \mathrm{a}^{*}\right.$ and $\left.1.5 \mathrm{a}^{*}\right)$ and for three magnetic field intensity $(\gamma=0,0.4$ and 0.8$)$. The impurity donor is placed in the IQD layer centre $a_{0}=\frac{R_{1}+R_{2}}{2}$. We note that the diamagnetic susceptibility increases as a function of the magnetic field (the absolute value of the diamagnetic susceptibility $\mid \chi_{\text {dia }} /$ decreases as a function of the magnetic field). This increase is not linear especially for thin layer, and it is more pronounced for large layer than thin layer. The diamagnetic susceptibility decreases as $R_{2}$ increases and presents a minimum corresponding to a certain critical values wich is in good agreement with the Ref [21] and $\square$ tends to the limit representing the two-dimensional case when the ratio $\left(R_{1} / R_{2}\right)$ tends to 1 .The wave function cannot spread in the barrier regions and the electronimpurity distance decreases which affect the diamagnetic susceptibility. In Figure 5, we present the diamagnetic susceptibility as a function of impurity donor position for fixed IQD inner radius $\left(\mathrm{R}_{1}=0.5 \mathrm{a}^{*}\right.$ and $\left.\mathrm{R}_{1}=2 \mathrm{a}^{*}\right)$. Here the outer radius is $\mathrm{R}_{2}$ $=3 \mathrm{a}^{*}$. We remark that when the magnetic field increase the diamagnetic susceptibility increases. It is found that the magnetic field effect is more pronounced around a critical value of the position of the impurity $\left(\mathrm{a}_{0}\right)$. Theses effect decreases progressively as the impurity moves towards the spherical layer extremities for all confinement regimes. The susceptibility increases as the magnetic field increases and this magnetic field effect is more pronounced when the donor is placed near the extremities of spherical layer. We also find the same value of diamagnetic susceptibility $\chi_{\text {dia }}$ for HQD and IQD in the case $R_{1}=0$ and $R_{2}=1 a^{*}\left(\chi_{\text {dia }}=-0.16 \mathrm{a} . \mathrm{u}\right)$ , $\mathrm{R}_{1}=0 \mathrm{a}^{*}$ and $\mathrm{R}_{2}=1.5 \mathrm{a}^{*}\left(\chi_{\text {dia }}=-0.32 \mathrm{a} \cdot \mathrm{u}\right)$.

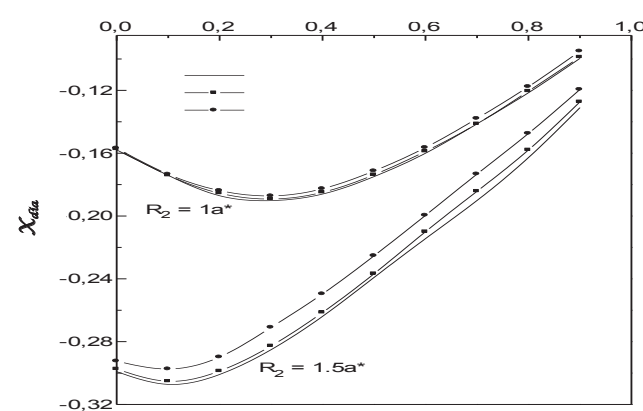

Figure 4 . The Diamagnetic Susceptibility versus the ratio $R_{1} / R_{2}$ for two values of the outer radius $\left(\mathrm{R}_{2}=1 \mathrm{a}^{*}\right.$ and $\left.\mathrm{R}_{2}=1.5 \mathrm{a}^{*}\right)$ and for three values of the magnetic field $(\gamma=0,0.4$ and 0.8$)$.

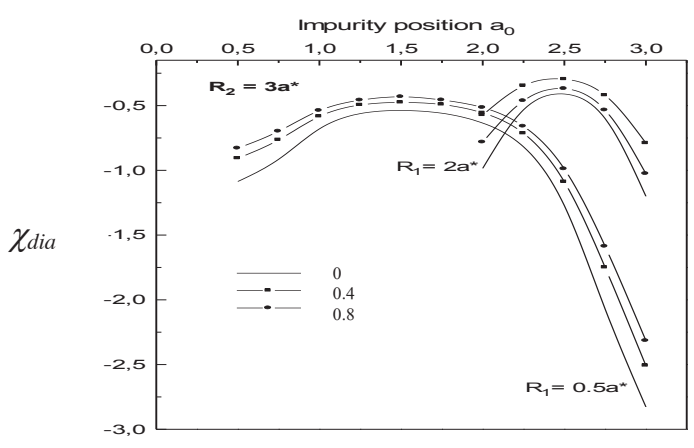

Fig. 5 . The Diamagnetic Susceptibility with three values of magnetic field $(\gamma=0,0.4$ and 0.8$)$ as a function of the impurity position for different values of the inner radius $\mathrm{R}_{1}=0.5 \mathrm{a}^{*}$ and $2 \mathrm{a}^{*}\left(\mathrm{R}_{2}=3 \mathrm{a}^{*}\right)$.

\section{Conclusion}

In this work, the impurity position and the external magnetic field effects on the diamagnetic susceptibility of a donor placed inside a Spherical IQD are investigated. The results show that the diamagnetic susceptibility is strongly influenced by the magnetic field, the donor position and the dimensional nanostructure. The magnetic field effect is more appreciable especially for large spherical layers. The diamagnetic susceptibility presents a maximum corresponding to a critical position value $a_{0}=\left(R_{1}+R_{2}\right) / 2$ where $\mathrm{R}_{1}\left(\mathrm{R}_{2)}\right.$ is the inner (outer) radius. This maximum is observed for strong confinement regime and when the impurity is located in the spherical layer center (off-center).

\footnotetext{
*Corresponding author: izorkani@hotmail.com
} 


\section{Acknowledgements}

Two of the authors, I. Zorkani, and S. Janati Edrissi would like to thank the Abdus Salam International Centre for Theoretical Physics .

\section{References}

1. Q.H. Zhong, and C.H. Liu, Thin Solid Films. (2008), 516, 3405.

2. J.A. Brum, Solid State Commun. (1985), 54, 179.

3. F. Hatami, and O. Bierwagen, (2011), 523.4. L. Tsakalakos, Materials Sci. Eng. R (2008), 62, 175

5. Z.X. Gui, and Guiguang, Gao, F. Microelectron. J. (2007), 38, 447.

6. M. Cristea, and E.C. Niculescu, Physics Letters A (2013), 377, 1221-.

7. V.A. Fonoberov, Pokatilova, and E.P, V.M. Fominc, J.T. Devreese, Physica E (2005), 26, 63.

8. V.A. Harutyunyan, Physica E. (2007), 39, 37.

9. Yinan Fang, Meng Xiao and Duanzheng Yao, Physica E. (2010), 42, 2178-2183.

10. I. Zorkani, K. Rahmani, M. El Yahyaoui, and A. Seddik,.D. Phys.Low-Dim.Struct. (2003), 11, 85.
11. H.El Ghazi, A.Jorio, and I. Zorkani, Physica B (2013), 426, 155-157.

12. A.Talbi, E.Feddi, A.Oukerroum, E. Assaid, and M. Addou Superlatt. Microstruct.(2015), 85, 581 .

13. H. El Ghazi, A. Jorio, and I. Zorkani, Physica B. (2013), 422, 47.

14. H. El Ghazi, A. Jorio, and I. Zorkani, Physica B. (2013), 427, 106.

15. K. Rahmani, and I. Zorkani, Moroccan. J. Condens. Mater Society, (2009), 11, 35.

16. A. Salmogli and A. J. Rostami, Nanopart Res., Springer science, ( 2010).

17. A. Mmadi, and I. Zorkani, K. Rahmani, and A. Jorio, The African Review of Physics. (2013) 8, 219.

18. A. Mmadi, K. Rahmani, I . Zorkani, and A. Jorio, Superlatt. Microstruct. (2013), 57, 27.

19. S. Janati.Edrissi,K. Rahmani, I. Zorkani, A. Mmadi, A. Jorio, IOP Conf.ser:Mater.Scl.Eng. (2017), 186.

20. Kittel, C. Introduction to Solid State Physics, seventh ed., Wiley, New York, (1998).

21. K. Rahmani, I. Zorkani, and A. Jorio, Phys.Scr. (2011), 83, 035701.

*Corresponding author: izorkani@hotmail.com 